\title{
Article \\ Taking the Convergent Close-Coupling Method beyond Helium: The Utility of the Hartree-Fock Theory
}

\author{
Igor Bray $^{1, *(\mathbb{D})}$, Xavier Weber ${ }^{1} \mathbb{D}$, Dmitry V. Fursa ${ }^{1} \mathbb{D}$, Alisher S. Kadyrov ${ }^{1} \mathbb{D}$, Barry I. Schneider $^{2} \mathbb{D}^{\mathbb{D}}$, \\ Sudhakar Pamidighantam ${ }^{3}$ (D), Maciej Cytowski ${ }^{4}(\mathbb{D})$ and Anatoli S. Kheifets ${ }^{5}$ (D)
}

1 Curtin Institute for Computation and Department of Physics and Astronomy, Curtin University, Perth, WA 6845, Australia; xavier.weber@student.curtin.edu.au (X.W.); D.Fursa@curtin.edu.au (D.V.F.); A.Kadyrov@curtin.edu.au (A.S.K.);

2 National Institute of Standards and Technology, Gaithersburg, MD 20899, USA; barry.schneider@nist.gov

3 Cyberinfrastructure Integration Research Center, Department of Chemistry, Indiana University, Bloomington, IN 47408, USA; pamidigs@iu.edu

4 Pawsey Supercomputing Centre, 1 Bryce Ave., Kensington, WA 6151, Australia; maciej.cytowski@pawsey.org.au

5 Research School of Physics, The Australian National University, Canberra, ACT 2601, Australia; a.kheifets@anu.edu.au

* Correspondence: I.Bray@curtin.edu.au

check for updates

Citation: Bray, I.; Weber, X.; Fursa, D.V.; Kadyrov, A.S.; Schneider, B.I.; Pamidighantam, S.; Cytowski, M.; Kheifets, A.S. Taking the Convergent Close-Coupling Method beyond Helium: The Utility of the

Hartree-Fock Theory. Atoms 2022, 10, 22. https://doi.org/10.3390/ atoms 10010022

Academic Editor: Grzegorz Piotr Karwasz

Received: 19 January 2022

Accepted: 5 February 2022

Published: 11 February 2022

Publisher's Note: MDPI stays neutral with regard to jurisdictional claims in published maps and institutional affiliations.

Copyright: () 2022 by the authors Licensee MDPI, Basel, Switzerland. This article is an open access article distributed under the terms and conditions of the Creative Commons Attribution (CC BY) license (https:// creativecommons.org/licenses/by/ $4.0 /)$.

\begin{abstract}
The convergent close-coupling (CCC) method was initially developed to describe electron scattering on atomic hydrogen and the hydrogenic ions such as $\mathrm{He}^{+}$. The latter allows implementation of double photoionization (DPI) of the helium atom. For more complex single valence-electron atomic and ionic targets, the direct and exchange interaction with the inner electron core needs to be taken into account. For this purpose, the Hartree-Fock (HF) computer codes developed in the group of Miron Amusia have been adapted. In this brief review article, we demonstrate the utility of the HF technique by examples of electron scattering on $\mathrm{Li}$ and the DPI of the $\mathrm{H}^{-}$and $\mathrm{Li}^{-}$ions. We also discuss that modern-day computer infrastructure allows the associated CCC code, and others, to be readily run directly via the Atomic, Molecular and Optical Science Gateway.
\end{abstract}

Keywords: electron-atom scattering; atomic photoionization; many-electron correlation; electronic structure

PACS: $32.80 . \mathrm{Rm} ; 32.80 . \mathrm{Fb} ; 42.50 . \mathrm{Hz}$

arXiv: 2108.02392

\section{Introduction}

Collisions between particles on the atomic scale are ubiquitous throughout the universe. Our interest is in the collisions of fundamental particles such as electrons, positrons, photons, and protons with atoms and molecules. The field relies on strong interactions between experimental and theoretical approaches to the collision problems. The expectation is that experiments provide benchmark measurements for theorists to test their models, and when deemed sufficiently accurate the models provide extensive data for use in applications. The latter include astrophysics, fusion, lighting, nanolithography, and medical imaging and therapy. Accordingly, it is of great concern whenever there are substantial discrepancies between theory and experiment that are not understood. One such case was the discrepancy for the angular correlation parameters in the fundamental Coulomb three-body collision problem of e-H excitation of the $2 p$ state $[1,2]$.

From the theoretical side, the difficulties associated with calculating e- $\mathrm{H}$ scattering relate to the fact that the target has a countably infinite number of discrete states, an uncountably infinite continuum, and that the Coulomb interaction extends to infinite distances for charged particles. The convergent close-coupling (CCC) method $[3,4]$ was developed 
in response to the abovementioned discrepancy by providing a systematic and mathematically rigorous approach to the underlying computational difficulties. By expanding the target wave-functions in a truncated complete Laguerre basis the target is represented with a finite number of target states $N$. Furthermore, due to the exponential falloff of the basis, the interactions do not extend to infinite distances. The problem is then reduced to obtaining convergence in the scattering amplitudes of interest with increasing $N$. Despite demonstrating such convergence, the CCC method was unable to resolve the discrepancy with the experiment, and yielded results similar to other sophisticated theoretical approaches to the problem [4]. However, it was able to yield excellent agreement with the measurements of the total ionization cross section and its spin asymmetry [5], which is a particularly stringent test of any theory.

While atomic hydrogen is the ideal starting point for testing any electron-atom scattering theory, it is not ideal from the experimental point of view. Alkali atoms such as $\mathrm{Li}$ and $\mathrm{Na}$ are easier to prepare in the laboratory, and yet their chemistry is very similar to that of $\mathrm{H}$, having just one valence electron. This allowed for some very accurate measurements of e-Li [6-8], e-Na [9-12], and e-K [13-15] scattering systems. So the next stage in trying to understand the discrepancy for the e-H system was to extend the CCC method to the alkali atoms. The group of Miron Amusia generously provided the Hartree-Fock computational code $[16,17]$ for a self-consistent treatment of the core electrons. This allowed the reduction of the electron-alkali atom collision problem to also be a Coulomb three-body problem, albeit with some more complicated nonlocal potentials [18]. The Hartree-Fock code also enabled the extension of the two-electron CCC code [19] to quasi two-electron targets such as alkaline earth metals $[20,21]$. Reviews of the development of the CCC method, including application to differential ionization, are available [22-25].

Unlike the case of the e-H system, the agreement between the CCC calculations and experiment for the electron-alkali collisions was spectacular, so much so, that the e- $\mathrm{H}$ collision system was remeasured by other groups, who found excellent agreement with the CCC calculations $[26,27]$. Subsequently, errors in the original measurements were identified [28].

A similar development track was taken by DPI on He, where the final state is determined from the e- $\mathrm{He}^{+}$collision system. Here too. theoretical predictions did not always agree with the initial experiment. Mergel et al. [29] measured the DPI of He using circularly polarized light. However, the corresponding CCC calculations did not yield agreement with the measurements [30]. Subsequently, Achler et al. [31] revisited the experiment and found excellent agreement with the CCC calculations. The CCC method was then extended to describe double ionization of He by electron impact (the so-called $(e, 2 e)$ reaction $[32,33]$ ). Then an extension was made to the process of two-photon double ionization of $\mathrm{He}$ [34]. The utility of the HF theory was instrumental to describe the valence-shell DPI of alkalineearth metal atoms [35]. These calculations were later found in good agreement with experiments [36]. Most recently, the CCC technique aided by the HF theory was applied to time-resolved atomic photoemission. The time delay in photoemission, expressed via the phase of the complex ionization amplitude, became experimentally accessible [37,38]. While the measurements are restricted at present to single active electron targets, theoretical predictions for two-electron targets have been made [39,40]. Most recently, photodetachment time delay was analyzed to reveal the implications of the fundamental threshold laws [41].

Here we shall demonstrate the agreement between theory and experiment by focusing on just the simplest electron-alkali collision system, that of e-Li scattering. This collision system is also a key component in calculating the DPI of $\mathrm{Li}^{-}$, as upon single or double photoionization the e-Li wave-function corresponds to the final state of $\mathrm{Li}^{-}$. The CCC computer codes utilize modern computational infrastructure including massive parallelism and GPU acceleration and are readily accessible for execution via the Atomic, Molecular and Optical Science Gateway, https: / / amosgateway.org (accessed on 4 February 2022) [42].

\section{Theory}

The details of the implementation of the CCC theory to electron scattering on quasi one-electron targets, such as the alkalis, have been given by Bray [18]. Briefly, the core- 
electron wave-functions $\psi_{j}$ of target $T$ are solved for by utilizing the Self-consistent-Field Hartree-Fock (SCHF) equations [16]

$$
\left(K+V^{\mathrm{HF}}-\varepsilon_{j}\right) \psi_{j}(\boldsymbol{r})=0, \quad \psi_{j} \in T,
$$

where

$$
\begin{aligned}
V^{\mathrm{HF}} \psi_{j}(\boldsymbol{r})= & \left(-\frac{Z}{r}+2 \sum_{\psi_{j^{\prime}} \in T} \int d^{3} r^{\prime} \frac{\left|\psi_{j^{\prime}}\left(\boldsymbol{r}^{\prime}\right)\right|^{2}}{\left|\boldsymbol{r}-\boldsymbol{r}^{\prime}\right|}\right) \psi_{j}(\boldsymbol{r}) \\
& -\sum_{\psi_{j^{\prime}} \in T} \int d^{3} r^{\prime} \frac{\psi_{j^{\prime}}^{*}\left(\boldsymbol{r}^{\prime}\right) \psi_{j}\left(\boldsymbol{r}^{\prime}\right)}{\left|\boldsymbol{r}-\boldsymbol{r}^{\prime}\right|} \psi_{j^{\prime}}(\boldsymbol{r}) .
\end{aligned}
$$

The core-electron wave-functions are then used to define the Frozen-Core Hartree-Fock (FCHF) potential $V^{\mathrm{FC}}$ as

$$
\begin{aligned}
V^{\mathrm{FC}} \phi_{j}(\boldsymbol{r})= & \left(-\frac{Z}{r}+2 \sum_{\psi_{j^{\prime}} \in C} \int d^{3} r^{\prime} \frac{\left|\psi_{j^{\prime}}\left(\boldsymbol{r}^{\prime}\right)\right|^{2}}{\left|\boldsymbol{r}-\boldsymbol{r}^{\prime}\right|}\right) \phi_{j}(\boldsymbol{r}) \\
& -\sum_{\psi_{j^{\prime}} \in C} \int d^{3} r^{\prime} \frac{\psi_{j^{\prime}}^{*}\left(\boldsymbol{r}^{\prime}\right) \phi_{j}\left(\boldsymbol{r}^{\prime}\right)}{\left|\boldsymbol{r}-\boldsymbol{r}^{\prime}\right|} \psi_{j^{\prime}}(\boldsymbol{r}),
\end{aligned}
$$

where the notation $C$ indicates the set of frozen-core wave-functions. The target wavefunctions are then obtained from the effective one-electron Hamiltonian $K+V^{\mathrm{FC}}$ via

$$
\left(K+V^{\mathrm{FC}}-\epsilon_{j}\right) \phi_{j}(\boldsymbol{r})=0 .
$$

The eigenstates can be obtained directly [17] or via diagonalization in some suitable basis. In the CCC method we do both, with the utilization of the Laguerre basis, which yields negative-energy eigenstates and a discretization of the target continuum.

With all of the potentials $V$ defined, the close-coupling equations are formed in momentum space as coupled Lippmann-Schwinger equations directly for the transition amplitudes $\left\langle\boldsymbol{k}_{f} \phi_{f}\left|T_{S}\right| \phi_{i} \boldsymbol{k}_{i}\right\rangle$, as if the problem was a three-body one $[4,18]$

$$
\left\langle\boldsymbol{k}_{f} \phi_{f}\left|T_{S}\right| \phi_{i} \boldsymbol{k}_{i}\right\rangle=\left\langle\boldsymbol{k}_{f} \phi_{f}\left|V_{S}\right| \phi_{i} \boldsymbol{k}_{i}\right\rangle+\sum_{n=1}^{N} \int d^{3} k \frac{\left\langle\boldsymbol{k}_{f} \phi_{f}\left|V_{S}\right| \phi_{n} \boldsymbol{k}\right\rangle\left\langle\boldsymbol{k} \phi_{n}\left|T_{S}\right| \phi_{i} \boldsymbol{k}_{i}\right\rangle}{E^{(+)}-\epsilon_{n}-k^{2} / 2}
$$

where $S$ is the total electron spin, and $N$ is the number of Laguerre-based target states. We check for convergence in the required $\left\langle\boldsymbol{k}_{f} \phi_{f}\left|T_{S}\right| \phi_{i} \boldsymbol{k}_{i}\right\rangle$ by simply increasing $N$.

\section{Results}

\subsection{Electron-Lithium Excitation}

In the seminal publications of Bederson $[43,44]$, the idea of perfect scattering experiments was introduced. In such experiments, the maximum amount of the underlying scattering information is measured. In the case of P-state excitation, the angular correlation parameters discussed above, or the equivalent Stokes parameters [45], which may depend on total electron spin [46], complement the differential cross sections in providing the extra experimental information to compare with the theory. In the following three figures, we examine e-Li excitation of the $2 \mathrm{P}$ state by presenting spin-dependent and -weighted results for the differential cross sections (DCS) and the three Stokes parameters. For each total electron spin $S$, at every scattering angle, there are two independent scattering (complex) amplitudes resulting in four independent theoretical predictions. While experiment [8] is available only for the spin-weighted parameters, it can be thought of as perfect for the spin-weighted case.

In addition to presenting the CCC calculations, which are convergent in the treatment of both the target discrete and the continuous spectrum, the $\mathrm{CC}$ calculations are also 
presented. These are convergent in the treatment of just the discrete spectrum; hence, the differences between CCC and CC highlight the importance of the inclusion of the target continuum on the transition of interest.

In Figure 1, we present the results for $7 \mathrm{eV}$ e- $\mathrm{Li}$ excitation of the $2 \mathrm{P}$ state. We see that there is a substantial dependence of the results on the total electron spin $S$. The importance of the continuum is quite small at this energy. The agreement of the calculations with the experiment of Karaganov et al. [8] is quite extraordinary. Note that this experiment utilise the superelastic technique, which yields much better statistics than the traditional electron-photon coincidence experiments [1,2].
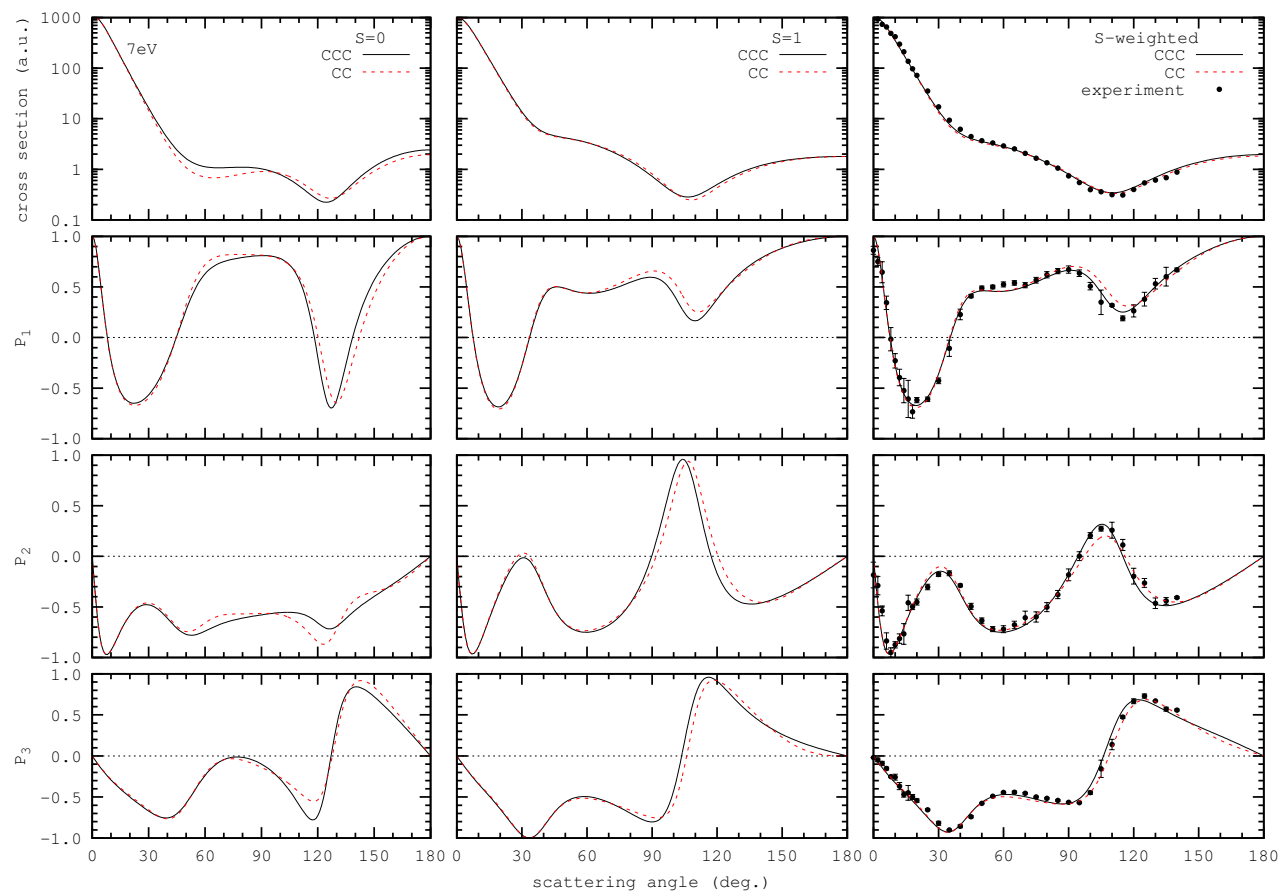

Figure 1. Differential cross section and (reduced) Stokes parameters for 7 eV electron-lithium $2 \mathrm{P}$ excitation. CCC results are convergent in the treatment of the Li discrete and continuous spectrum, whereas CC results are convergent in the treatment of the discrete spectrum only. Experiment is due to Karaganov et al. [8].

The results for $14 \mathrm{eV}$ e-Li excitation of the $2 \mathrm{P}$ state are presented in Figure 2. This time we see substantial differences between the CCC and CC calculations, though mostly for the $S=0$ DCS and Stokes parameters. These differences are much less visible when compared with the experiment for the spin-weighted Stokes parameters, where the agreement is again outstanding.

Lastly, the $22 \mathrm{eV}$ e- $\mathrm{Li}$ excitation of the $2 \mathrm{P}$ state is presented in Figure 3. At this energy the inclusion of the target continuum is clearly important for $S=0$, though this is less visible for the spin-weighted parameters. The agreement with the experiment is outstanding once more. It was such good agreement, first reported by [6], that others seriously questioned the accuracy of the corresponding e- $\mathrm{H} 2 \mathrm{P}$ excitation measurements. 

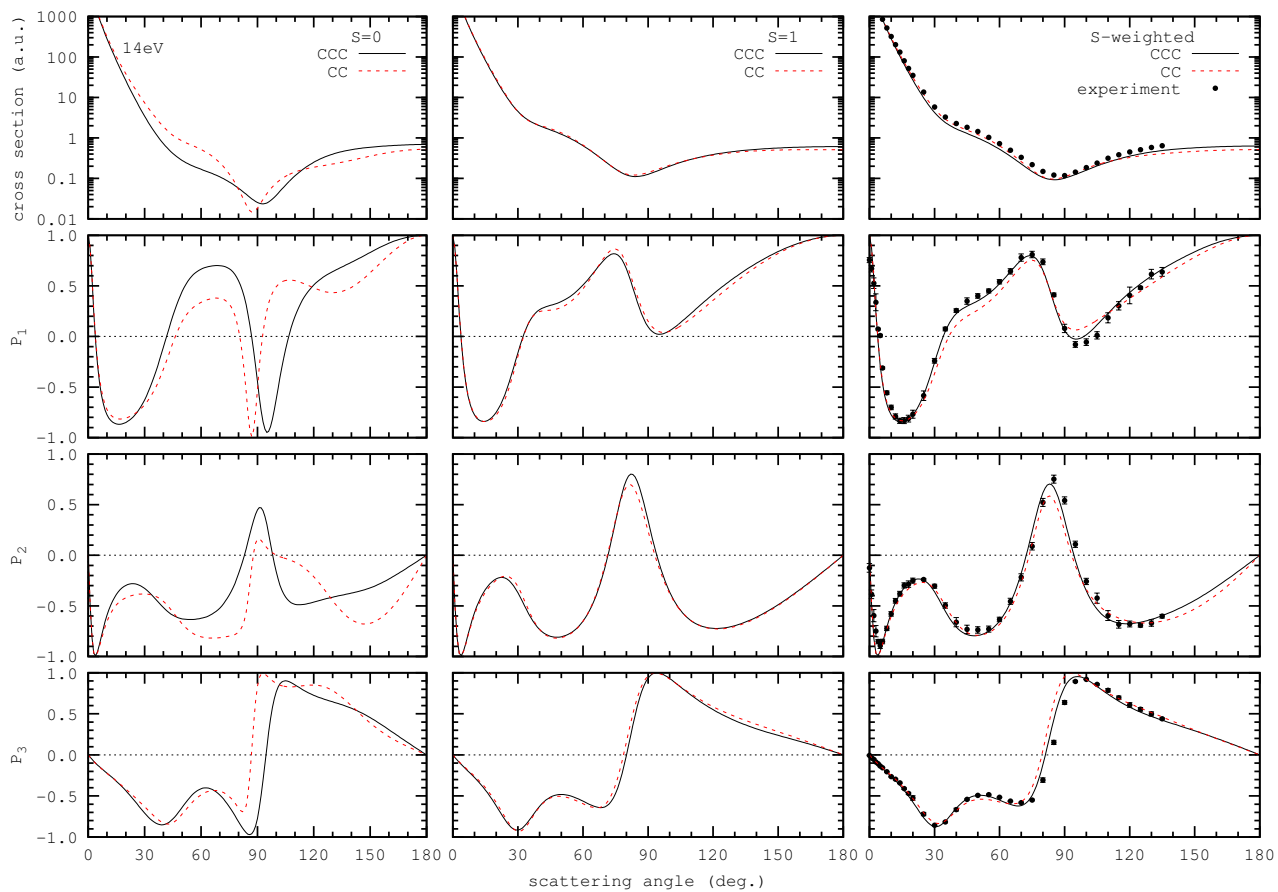

Figure 2. Same as for Figure 1, except for $14 \mathrm{eV}$.
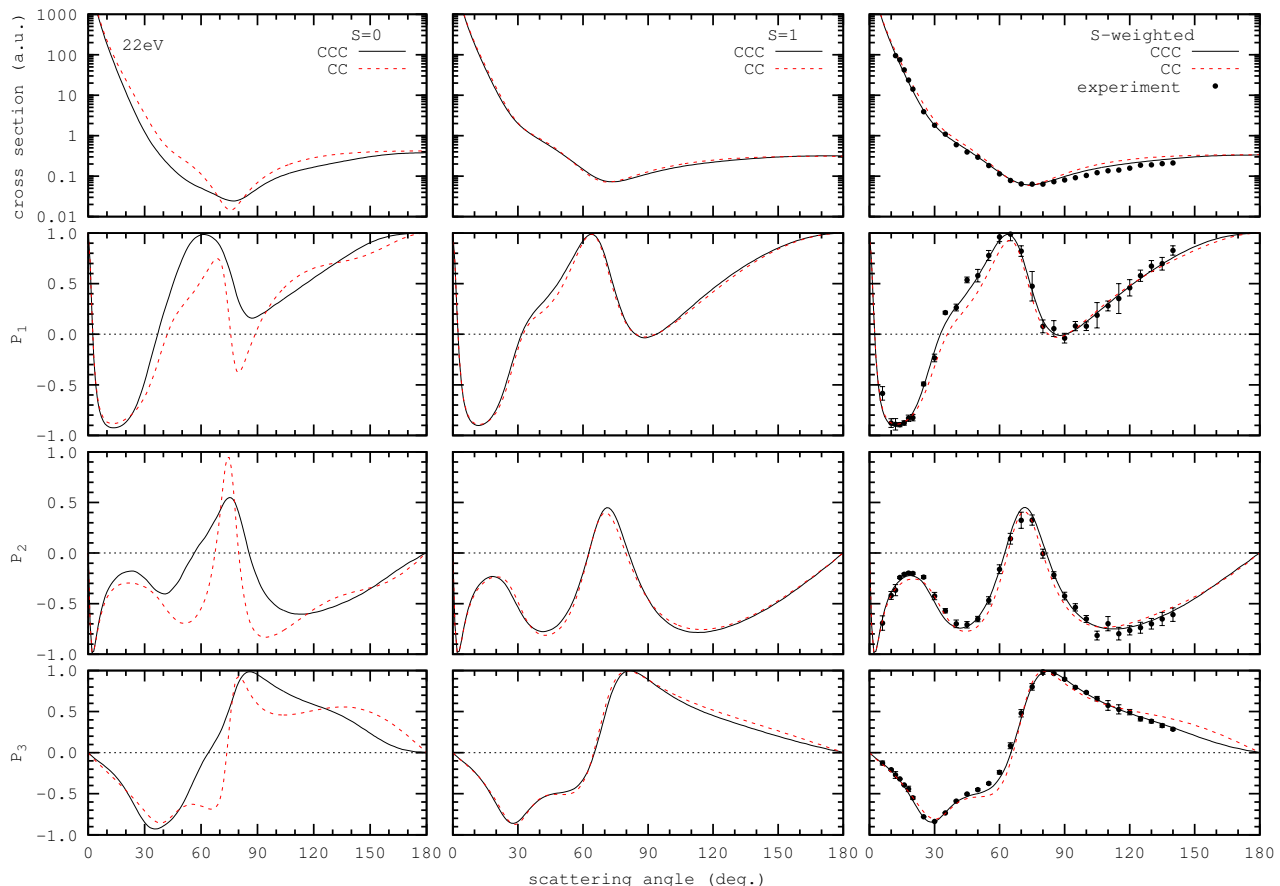

Figure 3. Same as for Figure 1, except for $22 \mathrm{eV}$.

\subsection{Double Photoionisation}

The knowledge of the scattering T-matrix allows expression of the two-electron dipole matrix element in the form of an integral [47]:

$$
\begin{aligned}
& \left\langle\Psi_{j}^{(-)}(\boldsymbol{k})|\mathcal{D}| \Psi_{0}\right\rangle=\left\langle\boldsymbol{k}^{(-)} \phi_{j}|\mathcal{D}| \Psi_{0}\right\rangle \\
& \quad+\sum_{i} \mathcal{L} d^{3} \kappa \frac{\left\langle\boldsymbol{k}^{(-)} \phi_{j}|T| \phi_{i} \boldsymbol{\kappa}^{(+)}\right\rangle\left\langle\boldsymbol{\kappa}^{(+)} \phi_{i}|\mathcal{D}| \Psi_{0}\right\rangle}{E-\varepsilon_{\boldsymbol{\kappa}}-\epsilon_{i}+i 0} .
\end{aligned}
$$


Here, the bare dipole matrix element $\left\langle\boldsymbol{k}^{(-)} \phi_{j}|\mathcal{D}| \Psi_{0}\right\rangle$ is calculated between the twoelectron initial state $\Psi_{0}$ and the final channels $\left\langle\boldsymbol{k}_{b}^{(-)} \phi_{j}\right|$. The initial state is expressed in the form of the multiconfiguration Hartree-Fock (MCHF) expansion

$$
\Psi_{0}\left(\boldsymbol{r}_{1}, \boldsymbol{r}_{2}\right)=\sum_{l=0}^{l_{\max }} \sum_{m=n}^{n_{\max }} C_{m l}\left|\psi_{m l}\left(\boldsymbol{r}_{1}\right) \psi_{m l}\left(\boldsymbol{r}_{2}\right):{ }^{1} S\right\rangle
$$

The MCHF orbitals $\psi_{m l}(\boldsymbol{r})$ are found in the frozen $1 s^{2}$ core in the case of $\mathrm{Li}^{-}(n=2)$, while this core is absent in the case of $\mathrm{H}^{-}(n=1)$. Only diagonal $m l^{2}$ terms are included in expansion (7), as is always the case for the closed-shell MCHF ground state. The coefficients $C_{m l}$ in the MCHF expansion (7) are found by using the multiconfiguration Dirac-Fock computer code [48].

The cross section of a two-electron transition, measured as a function of the photon energy $\omega$ and corresponding to a particular state $j$ of the remaining bound electron, is given by [49]:

$$
\sigma_{j}(\omega)=\frac{4 \pi^{2}}{\omega c} \sum_{m_{j}} d^{3} k\left|\left\langle\Psi_{j}^{(-)}(\boldsymbol{k})|\mathcal{D}| \Psi_{0}\right\rangle\right|^{2} \delta\left(\omega-E+E_{0}\right) .
$$

Here, $c \simeq 137$ is the speed of light in atomic units, while other fundamental constants are set to $e=m=\hbar=1$. The final channels are separated into single and double ionization according to the energy $\epsilon_{j}$, which is positive for the double ionized channels and negative for the singly ionized channels.

The phase of the ionization amplitude

$$
\delta_{j}(\boldsymbol{k})=\arg \left\langle\Psi_{j}^{(-)}(\boldsymbol{k})|\mathcal{D}| \Psi_{0}\right\rangle, \tau_{j}(\boldsymbol{k})=\partial \delta_{j}(\boldsymbol{k}) / \partial E
$$

is used to calculate the photoemission time delay $\tau_{j}(\boldsymbol{k})$ in the given emission direction as the phase derivative with respect to the photoelectron energy $E=k^{2} / 2$ (see Equation (S10) of [37]).

Our numerical results for photodetachment of $\mathrm{H}^{-}$and $\mathrm{Li}^{-}$are displayed in Figures 4 and 5, respectively. We select these two targets because the threshold laws manifest themselves particularly clearly in negative ions, which have a very simple spectrum and can support only a limited number of discrete excited states in comparison to an infinite number in their neutral atomic counterparts.

The photodetachment cross section (8) as well as the phase and the time delay (9) of $\mathrm{H}^{-}$near the $n=2$ threshold are presented in Figure 4. The top panel displays the partial photodetachment cross sections in various channels leaving the $\mathrm{H}$ atom in the ground $1 \mathrm{~s}$ and excited $2 s$ or $2 p$ states. The total cross section is compared with the experiment [50]. The sharp resonance below the $n=2$ threshold is due to autodetachment of a two-electron bound state into the ground state of the $\mathrm{H}$ atom (Feshbach resonance). We see that all four autodetachment channels have very similar cross sections. The time delay in the $n=2$ channels is strongly angular-dependent. Near the opening of the $n=2$ channels, it reaches very large negative values.

Figure 5 displays the set of $\mathrm{Li}^{-}$results analogous to that of $\mathrm{H}^{-}$shown in Figure 4 . The photodetachment cross section exhibited in the top panel of Figure 5 displays a very clear threshold cusp prescribed by the Wigner threshold law [51], which suppresses all the partial waves in the photoelectron wave packet in the newly opened channel except the $s$-wave. 

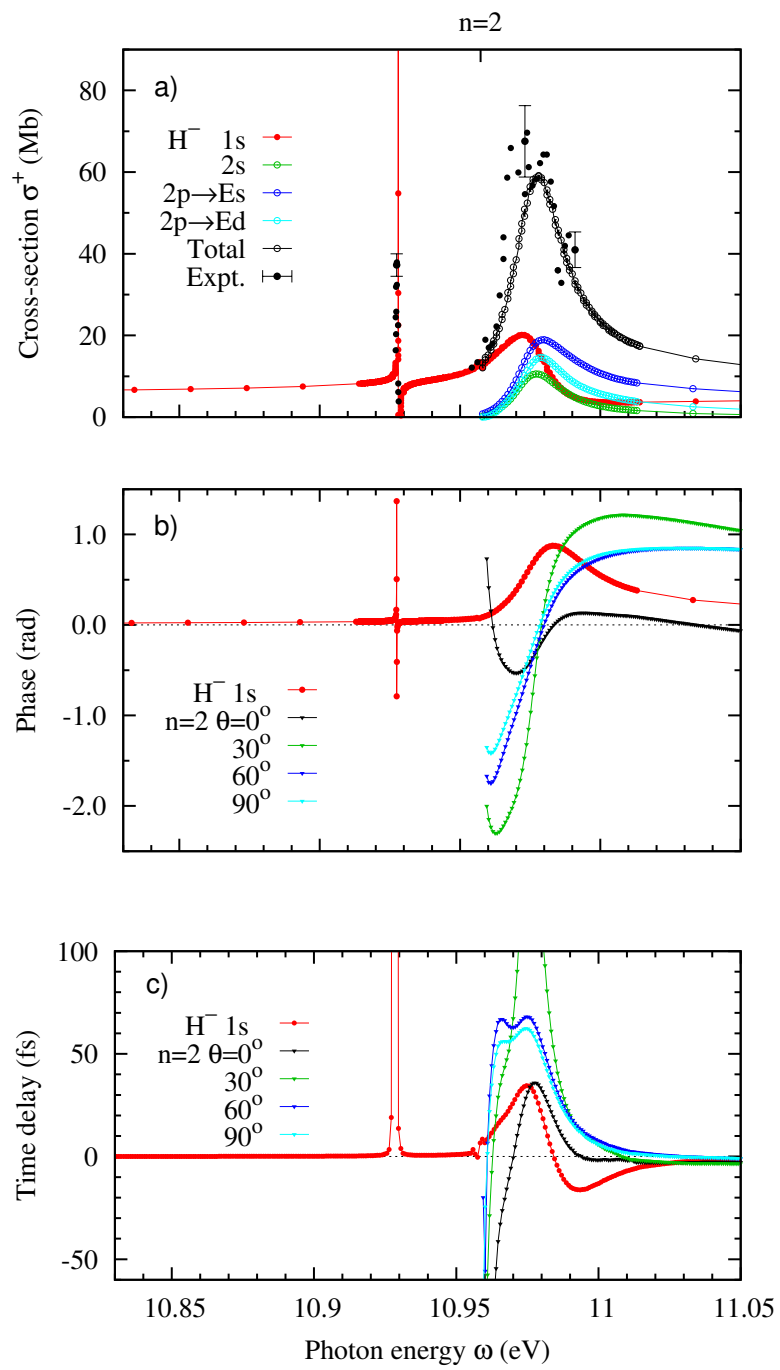

Figure 4. (Color online) (a) The cross section (8), (b) the phase of the transition amplitude, and (c) the time delay (9) of $\mathrm{H}^{-}$in various photodetachment channels near the $n=2$ threshold. The experiment [50] is displayed in the top panel.

In the Wigner theory, the cross section near the opening of a new channel can be parameterized as

$$
\sigma(E)=\sigma_{0}-2 A\left|E-E_{0}\right|^{1 / 2} \times\left\{\begin{array}{ll}
\sin ^{2} \delta_{0} & \text { for } E>E_{0} \\
\sin \delta_{0} \cos \delta_{0} & \text { for } E<E_{0}
\end{array} .\right.
$$

The scattering phase $\delta_{0}$ is rather small in $\mathrm{H}^{-}$, as the inelastic threshold is $3 / 4 \mathrm{Ry}$ above the photodetachment threshold. In contrast, in $\mathrm{Li}^{-}$, the inelastic $2 p$ threshold is only $2 \mathrm{eV}$ above the photodetachment threshold, and the phase $\delta_{0}$ is rather large. The Wigner Formula (10) predicts the falling cross section above the threshold, while it is always rising below the threshold, hence the cusp formation and the strong dominance of the $s$-wave in the inelastic channel of $\mathrm{Li}^{-}$. This dominance reduces dramatically the angular dependence of the phase and time delay. The latter is only weakly angular dependent in comparison with the $\mathrm{H}^{-}$ion. 

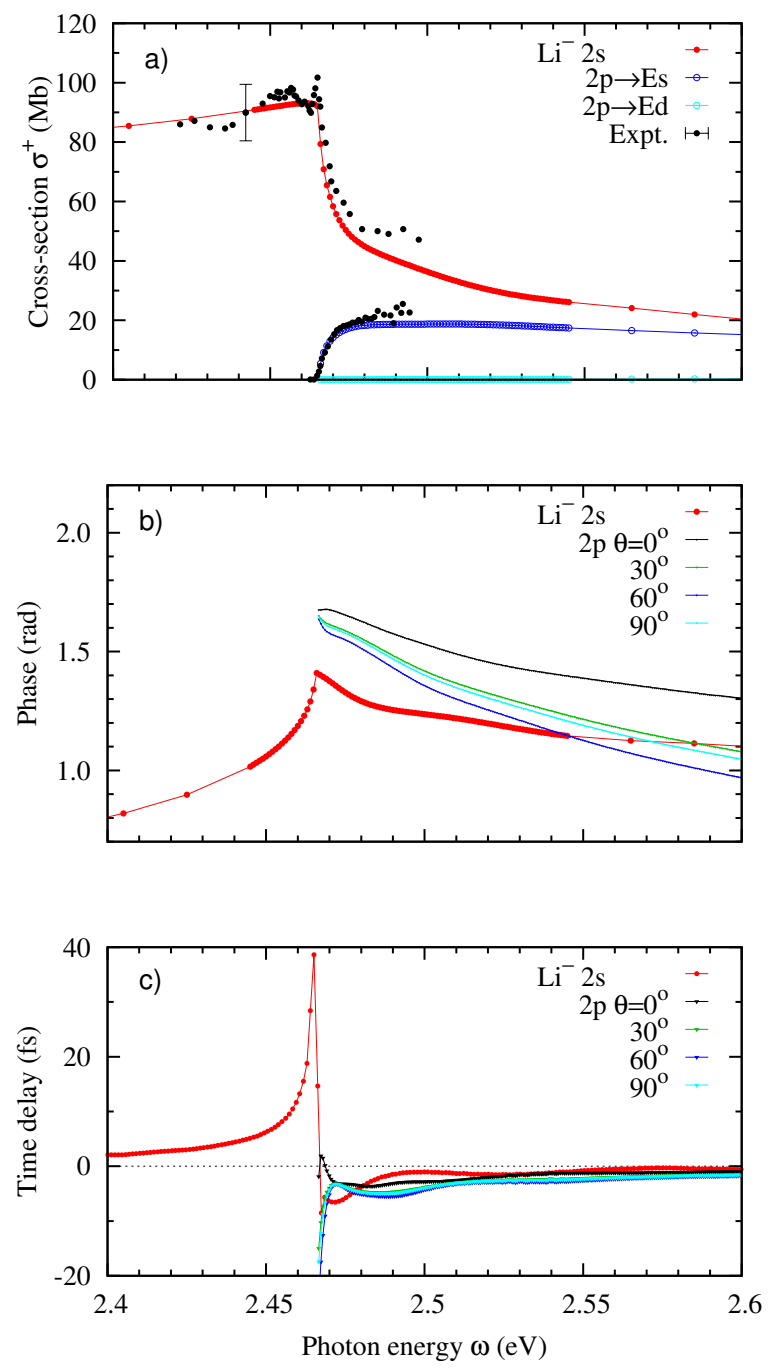

Figure 5. (Color online) (a) The cross section (8), (b) the phase of the transition amplitude, and (c) the time delay (9) of $\mathrm{H}^{-}$in various photodetachment channels near the $n=2$ threshold. Experimental cross section results $[52,53]$ are displayed in the top panel.

\subsection{Atomic, Molecular and Optical Science Gateway}

The CCC calculations presented here may be reproduced in a matter of minutes using the Atomic, Molecular and Optical Science (AMOS) Gateway https:/ / amosgateway.org (accessed on 4 February 2022), which is accessible to anyone who is able to authenticate via their own institution. The gateway is a sustainable community-oriented platform for enabling AMOS applications as a service for the AMOS community with intuitive interfaces. Several other AMOS codes are either available, or being made available, via this interface [42].

The gateway-accessible CCC code has been installed and compiled for efficient execution on the machines of the Extreme Science and Engineering Discovery Environment (XSEDE). The code has inbuilt hybrid MPI and OpenMP parallelization, as well as GPU acceleration. The latter has only been implemented recently [54] and continues to be a focus of development. Sample e-Li scattering inputs for the presented calculations are available. The gateway is constantly under development and we expect that more user-friendly interfaces will be built in due course.

\section{Conclusions}

We are grateful for our interactions with Miron Amusia and their group. Here, we have shown how the incorporation of the Self-Consistent Field and Frozen Core Hartree-Fock routines of the group have played a very important role in the development of the CCC 
method beyond atomic hydrogen and helium. Not only did this simply broaden the utility of the method but also helped resolve what previously seemed as intractable discrepancies between theory and experiment.

Author Contributions: Software, B.I.S., M.C. and S.P.; formal analysis, D.V.F. and A.S.K (Alisher S. Kadyrov); investigation, A.S.K. (Anatoli S. Kheifets); data curation, X.W.; writing-original draft preparation, I.B. All authors have read and agreed to the published version of the manuscript.

Funding: This research received no external funding.

Institutional Review Board Statement: Not applicable.

Informed Consent Statement: Not applicable.

Data Availability Statement: The numerical data reported in the present work are available on request from the author.

Acknowledgments: The authors gratefully acknowledge the Resources of National Computational Infrastructure facility (NCI Australia), The Pawsey Supercomputer Centre and the Extreme Science and Engineering Discovery Environment (XSEDE), which is supported by National Science Foundation, USA. The Curtin authors are supported by the Australian Research Council.

Conflicts of Interest: The authors declare no known conflict of interest.

\section{References}

1. Weigold, E.; Frost, L.; Nygaard, K.J. Large-angle electron-photon coincidence experiment in atomic hydrogen. Phys. Rev. A 1980, 21, 1950-1954. [CrossRef]

2. Williams, J.F. Electron-photon angular correlations from the electron impact excitation of the $2 \mathrm{~s}$ and $2 \mathrm{p}$ electronic configurations of atomic hydrogen. J. Phys. B At. Mol. Opt. Phys. 1981, 14, 1197-1217. [CrossRef]

3. Bray, I.; Stelbovics, A.T. Explicit demonstration of the convergence of the close-coupling method for a Coulomb three-body problem. Phys. Rev. Lett. 1992, 69, 53-56. [CrossRef]

4. Bray, I.; Stelbovics, A.T. Convergent close-coupling calculations of electron-hydrogen scattering. Phys. Rev. A 1992, 46, 6995-7011. [CrossRef] [PubMed]

5. Bray, I.; Stelbovics, A.T. Calculation of the total ionization cross section and spin asymmetry in electron-hydrogen scattering. Phys. Rev. Lett. 1993, 70, 746-749. [CrossRef] [PubMed]

6. Karaganov, V.; Bray, I.; Teubner, P.J.O.; Farrell, P. Super-elastic electron scattering on lithium. Phys. Rev. A 1996, 54, R9-R12. [CrossRef]

7. Karaganov, V.; Bray, I.; Teubner, P.J.O. Super-elastic electron-lithium scattering at 7 and 14 eV. J. Phys. B At. Mol. Opt. Phys. 1998, 31, L187-L191. [CrossRef]

8. Karaganov, V.; Bray, I.; Teubner, P.J.O. Electron scattering from optically pumped lithium atoms. Phys. Rev. A 1999, 59, $4407-4417$. [CrossRef]

9. McClelland, J.J.; Kelley, M.H.; Celotta, R.J. Superelastic scattering of spin-polarized electrons from sodium. Phys. Rev. A 1989, 40, 2321-2329. [CrossRef]

10. Scholten, R.E.; Lorentz, S.R.; McClelland, J.J.; Kelley, M.H.; Celotta, R.J. Spin-resolved superelastic scattering from sodium at 10 and 40 eV. J. Phys. B At. Mol. Opt. Phys. 1991, 24, L653-L659. [CrossRef]

11. Lorentz, S.R.; Scholten, R.E.; McClelland, J.J.; Kelley, M.H.; Celotta, R.J. Spin-resolved elastic scattering of electrons from sodium below the inelastic threshold. Phys. Rev. Lett. 1991, 67, 3761. [CrossRef] [PubMed]

12. Scholten, R.E.; Shen, G.F.; Teubner, P.J.O. Superelastic electron scattering from sodium: Alignment and orientation parameters. J. Phys. B At. Mol. Opt. Phys. 1993, 26, 987. [CrossRef]

13. Stockman, K.A.; Karaganov, V.; Bray, I.; Teubner, P.J.O. Superelastic Electron Scattering from Potassium. J. Phys. B At. Mol. Opt. Phys. 1998, 31, L867-L872. [CrossRef]

14. Stockman, K.A.; Karaganov, V.; Bray, I.; Teubner, P.J.O. Electron impact excitation of the $4^{2} \mathrm{P}$ state in potassium at $54.4 \mathrm{eV}$ : Differential cross section, alignment and orientation parameters. J. Phys. B At. Mol. Opt. Phys. 1999, 32, 3003-3013. [CrossRef]

15. Stockman, K.A.; Karaganov, V.; Bray, I.; Teubner, P.J.O. Electron scattering from laser excited states of potassium from 3 to $100 \mathrm{eV}$. J. Phys. B At. Mol. Opt. Phys. 2001, 34, 1105-1114. [CrossRef]

16. Chernysheva, L.V.; Cherepkov, N.A.; Radojevic, V. Self-consistent field Hartree-Fock program for atoms. Comp. Phys. Comm. 1976, 11, 57. [CrossRef]

17. Chernysheva, L.V.; Cherepkov, N.A.; Radojevic, V. Frozen core Hartree-Fock program for atomic discrete and continuous states. Comp. Phys. Comm. 1979, 18, 87-100. [CrossRef]

18. Bray, I. Convergent close-coupling method for the calculation of electron scattering on hydrogen-like targets. Phys. Rev. A 1994, 49, 1066-1082. [CrossRef]

19. Fursa, D.V.; Bray, I. Calculation of electron-helium scattering. Phys. Rev. A 1995, 52, 1279-1298. [CrossRef]

20. Fursa, D.V.; Bray, I. Convergent close-coupling calculations of electron-beryllium scattering. J. Phys. B At. Mol. Opt. Phys. 1997, 30, L273-L277. [CrossRef] 
21. Fursa, D.V.; Bray, I. Convergent close-coupling calculations of electron scattering on helium-like atoms and ions; electronberyllium scattering. J. Phys. B At. Mol. Opt. Phys. 1997, 30, 5895-5913. [CrossRef]

22. Bray, I.; Stelbovics, A.T. Calculation of Electron Scattering on Hydrogenic Targets. In Advances In Atomic, Molecular, and Optical Physics; Academic Press: Cambridge, MA, USA, 1995; Volume 35, pp. 209-254. [CrossRef]

23. Fursa, D.V.; Bray, I. TOPICAL REVIEW: Convergent close-coupling calculations of electron-helium scattering. J. Phys. B At. Mol. Opt. Phys. 1997, 30, 757-785. [CrossRef]

24. Bray, I.; Fursa, D.V.; Kadyrov, A.S.; Stelbovics, A.T.; Kheifets, A.S.; Mukhamedzhanov, A.M. Electron- and photon-impact atomic ionisation. Phys. Rep. 2012, 520, 135-174. [CrossRef]

25. Bray, I.; Abdurakhmanov, I.B.; Bailey, J.J.; Bray, A.W.; Fursa, D.V.; Kadyrov, A.S.; Rawlins, C.M.; Savage, J.S.; Stelbovics, A.T.; Zammit, M.C. Convergent close-coupling approach to light and heavy projectile scattering on atomic and molecular hydrogen. J. Phys. B At. Mol. Opt. Phys. 2017, 50, 202001. [CrossRef]

26. Yalim, H.; Cvejanovic, D.; Crowe, A. 1s-2p excitation of atomic hydrogen by electron impact studied using the angular correlation technique. Phys. Rev. Lett. 1997, 79, 2951-2954. [CrossRef]

27. O'Neill, R.W.; van der Burgt, P.J.M.; Dziczek, D.; Bowe, P.; Chwirot, S.; Slevin, J.A. Polarization correlation measurements of electron impact excitation of $\mathrm{H}(2 \mathrm{p})$ at $54.4 \mathrm{eV}$. Phys. Rev. Lett. 1998, 80, 1630-1633. [CrossRef]

28. Williams, J.F.; Mikosza, A.G. Atomic hydrogen revisited: Electron impact excitation to H(2p) at 54 eV. J. Phys. B At. Mol. Opt. Phys. 2006, 39, 4113-4122. [CrossRef]

29. Mergel, V.; Achler, M.; Dörner, R.; Khayyat, K.; Kambara, T.; Awaya, Y.; Zoran, V.; Nyström, B.; Spielberger, L.; McGuire, J.H.; et al. Helicity Dependence of the Photon-Induced Three-Body Coulomb Fragmentation of Helium Investigated by Cold Target Recoil Ion Momentum Spectroscopy. Phys. Rev. Lett. 1998, 80, 5301-5304. [CrossRef]

30. Kheifets, A.S.; Bray, I. Calculation of circular dichroism in helium double photoionization. Phys. Rev. Lett. 1998, 81, 4588-4591. [CrossRef]

31. Achler, M.; Mergel, V.; Spielberger, L.; Dorner, R.; Azuma, Y.; Schmidt-Böcking, H. Photo double ionization of He by circular and linear polarized single-photon absorption. J. Phys. B At. Mol. Opt. Phys. 2001, 34, 965-981. [CrossRef]

32. Lahmam-Bennani, A.; Duguet, A.; Gaboriaud, M.N.; Taouil, I.; Lecas, M.; Kheifets, A.S.; Berakdar, J.; Cappello, C.D. Complete experiments for the double ionisation of He: (e,3e) cross sections at $1 \mathrm{keV}$ impact energy and small momentum transfer. J. Phys. B At. Mol. Opt. Phys. 2001, 34, 3073-3087. [CrossRef]

33. Sakhelashvili, G.; Dorn, A.; Höhr, C.; Ullrich, J.; Kheifets, A.S.; Lower, J.; Bartschat, K. Triple Coincidence (e, $\gamma 2 e$ ) Experiment for Simultaneous Electron Impact Ionization Excitation of Helium. Phys. Rev. Lett. 2005, 95, 033201. [CrossRef] [PubMed]

34. Kheifets, A.S.; Ivanov, I.A. Convergent close-coupling calculations of two-photon double ionization of helium. J. Phys. B At. Mol. Opt. Phys. 2006, 39, 1731-1742. [CrossRef]

35. Kheifets, A.S.; Bray, I. Valence-shell double photoionization of alkaline-earth-metal atoms. Phys. Rev. A 2007, 75, 042703. [CrossRef]

36. Wehlitz, R. Simultaneous emission of multiple electrons from atoms and molecules using synchrotron radiation. Adv. At. Mol. Opt. Phys. 2010, 58, 1-76. [CrossRef]

37. Schultze, M.; Fiess, M.; Karpowicz, N.; Gagnon, J.; Korbman, M.; Hofstetter, M.; Neppl, S.; Cavalieri, A.L.; Komninos, Y.; Mercouris, T.; et al. Delay in Photoemission. Science 2010, 328, 1658-1662. [CrossRef]

38. Klünder, K.; Dahlström, J.M.; Gisselbrecht, M.; Fordell, T.; Swoboda, M.; Guénot, D.; Johnsson, P.; Caillat, J.; Mauritsson, J.; Maquet, A.; et al. Probing Single-Photon Ionization on the Attosecond Time Scale. Phys. Rev. Lett. 2011, 106, 143002. [CrossRef]

39. Kheifets, A.S.; Ivanov, I.A.; Bray, I. Timing analysis of two-electron photoemission. J. Phys. B At. Mol. Opt. Phys. 2011, 44, 101003. [CrossRef]

40. Kheifets, A.S.; Bray, A.W.; Bray, I. Attosecond Time Delay in Photoemission and Electron Scattering near Threshold. Phys. Rev. Lett. 2016, 117, 143202. [CrossRef]

41. Kheifets, A.S.; Bray, I. Time delay in two-electron photodetachment and tests of fundamental threshold laws. Phys. Rev. Res. 2021, 3, 043017. [CrossRef]

42. Schneider, B.I.; Bartschat, K.; Zatsarinny, O.; Hamilton, K.R.; Bray, I.; Scrinzi, A.; Martin, F.; Gonzalez Vasquez, J.; Tennyson, J.; Gorfinkiel, J.D.; et al. Atomic and Molecular Scattering Applications in an Apache Airavata Science Gateway. In Practice and Experience in Advanced Research Computing; PEARC'20, Association for Computing Machinery: New York, NY, USA, 2020; pp. 270-277. [CrossRef]

43. Bederson, B. Perfect scattering experiment I. Comments At. Mol. Phys. 1969, 1, 41.

44. Bederson, B. Perfect scattering experiment II. Comments At. Mol. Phys. 1969, 1, 65.

45. Andersen, N.; Gallagher, J.W.; Hertel, I.V. Collisional alignment and orientation of atomic outer shells. Phys. Rep. 1988, 165, 1-188. [CrossRef]

46. Andersen, N.; Bartschat, K.; Broad, J.T.; Hertel, I.V. Collisional alignment and orientation of atomic outer shells. III. Spin-resolved excitation. Phys. Rep. 1997, 279, 251-396. [CrossRef]

47. Kheifets, A.S.; Bray, I. Calculation of double photoionization of helium using the convergent close-coupling method. Phys. Rev. A 1996, 54, R995-R997. [CrossRef]

48. Dyall, K.G.; Grant, I.P.; Johnson, C.T.; Parpia, F.P.; Plummer, E.P. GRASP: A general-purpose relativistic atomic structure program. Comp. Phys. Comm. 1989, 55, 425-456. [CrossRef]

49. Amusia, M.Y. Atomic Photoeffect; Plenum Press: New York, NY, USA, 1990.

50. Andersen, H.H.; Balling, P.; Kristensen, P.; Pedersen, U.V.; Aseyev, S.A.; Petrunin, V.V.; Andersen, T. Positions and Isotope Shifts of the $\mathrm{H}^{-}\left({ }^{1} P^{0}\right)$ Dipole Resonances below the $\mathrm{H}(n=2)$ Threshold. Phys. Rev. Lett. 1997, 79, 4770-4773. [CrossRef] 
51. Wigner, E.P. On the Behavior of Cross Sections Near Thresholds. Phys. Rev. 1948, 73, 1002-1009. [CrossRef]

52. Dellwo, J.; Liu, Y.; Pegg, D.J.; Alton, G.D. Near-threshold photodetachment of the Li ${ }^{-}$ion. Phys. Rev. A 1992, 45, 1544-1547. [CrossRef]

53. Dellwo, J.; Liu, Y.; Tang, C.Y.; Pegg, D.J.; Alton, G.D. Photodetachment cross sections for Li ${ }^{-}$. Phys. Rev. A 1992, 46, $3924-3928$. [CrossRef]

54. Bray, I.; Hayat, H.; Fursa, D.V.; Kadyrov, A.S.; Bray, A.W.; Cytowski, M. Calculations of electron scattering on H-like ions. Phys. Rev. A 2020, 101, 022703. [CrossRef] 